\title{
Formation of Insoluble and Colloidally Dispersed Tannic Acid Complexes in the Midgut Fluid of Manduca sexta (Lepidoptera: Sphingidae): An Explanation for the Failure of Tannic Acid to Cross the Peritrophic Envelopes of Lepidopteran Larvae
}

\author{
Raymond V. Barbehenn* and Michael M. Martin \\ Department of Biology, University of Michigan, Ann Arbor
}

\begin{abstract}
Magnesium and calcium ions, in concentrations comparable to those reported in the midgut fluids of lepidopteran larvae, bring about the precipitation of most of the tannic acid present in simple solutions buffered at $\mathrm{pH} 8.0$ and 10.0, but not at $\mathrm{pH}$ 6.5. In contrast, when tannic acid is added to Manduca sexta midgut fluid, less than $31 \%$ of the tannic acid added to the gut fluid is converted to a form that can be centrifuged into a pellet. The rest remains in the supernatant solution in the form of a colloidal suspension. Very little of the tannic acid, if any, remains in true solution. We suggest that the tannic acid-containing phase that is produced when tannic acid is added to midgut fluid is a complex multi-molecular aggregate of indefinite chemical composition, incorporating varying amounts of tannic acid, surface-active phospholipids, proteins, and polyvalent metal ions. On the basis of this study, we further suggest that the failure of tannins to diffuse across the peritrophic envelopes of lepidopteran larvae is a result of the capacity of the peritrophic envelope to act as a physical barrier to insoluble and colloidally dispersed particles, not the presence of substances in the matrix that strongly adsorb polyphenols or the presence of an extensive network of fixed anionic sites in the matrix that acts as an electrostatic barrier to the passage of polyphenolate anions. Arch. Insect Biochem. Physiol. 39:109117, 1998. ๑) 1998 Wiley-Liss, Inc.
\end{abstract}

Key words: tannins; complexation; peritrophic membrane; Lepidoptera

Abbreviations used: FITC = fluorescein isothiocyanate; HEPES
$=\mathrm{N}-\left[2\right.$-hydroxyethyl]piperazine- $\mathrm{N}^{\prime}[2$-ethanesulfonic acid];
HPLC = high performance liquid chromatography; MWCO =
molecular weight cutoff; $\mathrm{PE}=$ peritrophic envelope.

Contract grant sponsor: National Science Foundation; Contract grant number: BSR-8904043.
*Correspondence to: Raymond V. Barbehenn, Dept. of Biology, University of Michigan, Ann Arbor, MI 48109-1048.

Received 10 June 1998; Accepted 22 September 1998 


\section{INTRODUCTION}

The peritrophic envelope $\left(\mathrm{PE}^{*}\right)$, a non-cellular tubular sheath that lines the midgut of most insects (Peters, 1992), is permeable to most lowmolecular weight compounds (Peters, 1992) and biological polymers (Peters and Wiese, 1986; Santos and Terra, 1986; Barbehenn and Martin, 1995). An exception to this generalization is provided by the PEs of lepidopteran larvae, which are impermeable to tannins (Feeny, 1970; Barbehenn and Martin, 1992, 1994), even when the liquid phase of the midgut contents contains high concentrations of tannic acid $(5.0 \mathrm{mg} / \mathrm{ml})$ (Barbehenn, unpublished data). Impermeability to tannic acid is a characteristic of the PEs of caterpillars of species that are not tannin-tolerant or do not feed on tannin-containing foliage, e.g., Malacosoma disstria (Lasiocampidae) (Barbehenn and Martin, 1994) and Helicoverpa zea (Noctuidae) (Barbehenn and Lee, unpublished data), as well as those of tannin-adapted species, e.g., Orgyia leucostigma (Lymantriidae) (Barbehenn and Martin, 1992). Several plausible explanations for the failure of tannic acid to diffuse across the PEs of lepidopteran larvae have been advanced, only to be rejected when tested experimentally. For example, it has been proposed that adsorption on the $\mathrm{PE}$ is a mechanism by which tannins are contained within the endoperitrophic space of some Orthoptera (Bernays, 1981). However, our demonstration that less than $1 \%$ of the tannic acid present in a caterpillar's gut lumen is adsorbed by the $\mathrm{PE}$ demonstrates that this mechanism cannot explain the impermeability of the PE in Lepidoptera (Barbehenn and Martin, 1992). We proposed that anion exclusion, that is, the electrostatic repulsion between negatively charged polyphenolate ions and anionic sites in the matrix of the PE, might prevent diffusion of tannins (Barbehenn and Martin, 1994), but our subsequent demonstration that polyanionic dextran sulfates permeate the $\mathrm{PE}$ as readily as monoanionic dextrans ruled out that explanation (Barbehenn and Martin, 1997).

The purpose of this study is to test the hypothesis that tannins fail to cross the PEs of lepidopteran larvae because they exist in the midgut lumen in an undissolved state, either as an insoluble precipitate or a colloidal suspension, rather than in true solution. Tannins are known to form insoluble molecular aggregates with a plethora of chemicals, including proteins (Takechi and Tanaka, 1987; Hagerman and Klucher, 1986; Haslam et al., 1992; Stern et al., 1996; Baxter et al., 1997), polysaccharides (Cai et al., 1989, 1990; Haslam et al., 1992), lipids (Takechi and Tanaka, 1987; DeVeau and Schultz, 1992; Ikeda et al., 1992), alkaloids (Cai et al., 1990; Haslam et al., 1992), and polyvalent metal ions (Murdiati et al., 1991; Haslam et al., 1992; Slabbert, 1992; McDonald et al., 1996). In earlier studies we established that the PEs of lepidopteran larvae are effective barriers to FITC-dextrans with dimensions in excess of 20-30 nm (Barbehenn and Martin, 1995), while Santos and Terra (1986) and Ferriera et al. (1994) have proposed an even lower size exclusion limit, $8 \mathrm{~nm}$, for proteins. Large particles, such as bacteria and the virions of baculoviruses with dimensions greater than $60 \times 300 \mathrm{~nm}$, are totally contained within the endoperitrophic space (Derksen and Granados, 1988).

In this study we have addressed three questions: (1) Does tannic acid form a precipitate and/ or a colloidal suspension when added to a solution containing the monovalent and divalent cations that commonly occur in a caterpillar's midgut fluid? (2) Does tannic acid form a precipitate and/ or a colloidal suspension when added to the midgut fluid of the tomato hornworm, Manduca sexta (Lepidoptera: Sphingidae); and (3) What components of $M$. sexta gut fluid contribute to the formation of a precipitate or colloidal suspension when tannic acid is added to gut fluid? To address the first question we added tannic acid to buffered solutions ( $\mathrm{pH}$ 6.5, 8.0, 10.0) containing either sodium and potassium chloride or magnesium and calcium chloride at concentrations roughly comparable to those found in caterpillar midgut lumenal contents (Giordana and Sacchi, 1978; this study), centrifuged the mixture, and measured the amounts of tannic acid remaining in the supernatant layer. To address the second and third questions, we added tannic acid to $M$. sexta midgut fluid (or to gut fluid that had been deproteinized, defatted, or treated with Chelex 100 to remove magnesium and calcium ions), centrifuged the mixture, and determined what fraction of the tannic acid was precipitated and whether the tannic acid remaining in the supernatant layer would pass through an ultrafiltration membrane with a molecular weight cutoff of 100,000 Daltons.

We have chosen $M$. sexta as our experimental organism because it is a convenient source of the large quantitities of gut fluid necessary for 
the study. Since the PEs of all lepidopteran species that have been tested, tannin-sensitive as well as tannin-tolerant, have been found to be impermeable to tannic acid, the fact that this species does not normally consume tannin-containing foliage does not compromise our conclusions.

\section{MATERIALS AND METHODS Gut Fluid}

Gut fluid was obtained from $M$. sexta larvae reared from eggs (Carolina Biological Supply Co., Burlington, NC) through the penultimate instar on Douglas fir tussock moth artificial diet (Bioserv, Frenchtown, NJ) at $23^{\circ} \mathrm{C}$ under a 16 -h light:8-h dark photoperiod. During the first two days of the final instar, larvae were fed tomato (Lycopersicon esculentum) leaves. Early on the third day of the final instar, larvae were chilled $\left(-20^{\circ} \mathrm{C}, 13 \mathrm{~min}\right)$, and their guts were dissected from their bodies. Each gut was rinsed in distilled water and blotted dry. Guts were cut open lengthwise over the mouth of a $15-\mathrm{ml}$ screw-cap centrifuge tube, and the contents were allowed to drain into the tube. To maintain the gut fluid at 0\% oxygen (Johnson and Barbehenn, unpublished data), a gentle stream of nitrogen was directed into the mouth of the centrifuge tube, which was kept in crushed ice during the collection procedure. After collection of the gut contents, the tubes were centrifuged $\left(1,800 \mathrm{~g}, 30 \mathrm{~min}, 4^{\circ} \mathrm{C}\right)$ to remove large particles. Supernatant solutions were pooled, purged with nitrogen, capped and stored frozen $\left(-20^{\circ} \mathrm{C}\right)$. Just prior to use, gut fluid was thawed and re-centrifuged $\left(10,000 \mathrm{~g}, 10 \mathrm{~min}, 4.7^{\circ} \mathrm{C}\right)$.

\section{Concentrations of Metal lons in Gut Fluid}

Aliquots $(12 \mu \mathrm{l})$ of centrifuged gut fluid $(13,600 \mathrm{~g}, 5 \mathrm{~min})$, collected as described above from $M$. sexta or O. leucostigma larvae that had fed either on foliage ( $M$. sexta, tomato, $\mathrm{N}=4$; O. leucostigma, elm, $\mathrm{N}=2$ ) or Douglas fir tussock moth artificial diet $(M$. sexta, $\mathrm{N}=2 ; O$. leucostigma, $\mathrm{N}=4$ ), were diluted in $2.988 \mathrm{ml}$ of double-distilled water and analyzed for sodium, potassium, magnesium, calcium and iron using a Finnigan MAT ELEMENT ICP high resolution mass spectrometer.

\section{Interaction of Tannic Acid With Monovalent and Divalent Cations}

The interaction of tannic acid (Sigma, St. Louis, MO; lot 64F-0049) with monovalent alkali (sodium and potassium) and divalent alkaline earth (magnesium and calcium) cations was studied at three pHs. Buffers used were $0.05 \mathrm{M}$ HEPES (pH 6.5 and 8.0) and $0.05 \mathrm{M}$ glycine $(\mathrm{pH}$ 10.0). The precipitation of tannic acid by metal ions was studied in the following solutions: at $\mathrm{pH}$ 6.5, 8.0 and 10.0 in solutions containing either 3 $\mathrm{mM}$ sodium chloride and $160 \mathrm{mM}$ potassium chloride, or $21 \mathrm{mM}$ magnesium chloride and $32 \mathrm{mM}$ calcium chloride, and at $\mathrm{pH} 8.0$ and 10.0 in solutions containing either $0.51 \mathrm{mM}$ magnesium chloride and $0.33 \mathrm{mM}$ calcium chloride, or $2.6 \mathrm{mM}$ sodium chloride, $153.8 \mathrm{mM}$ potassium chloride, $0.51 \mathrm{mM}$ magnesium chloride, and $0.33 \mathrm{mM}$ calcium chloride. These concentrations were chosen because they are roughly comparable to the concentrations of these cations reported in the midgut fluids of some lepidopteran larvae (Giordana and Sacchi, 1978; this study). The same buffers without the added cations served as controls. Ascorbic acid ( $5 \mathrm{mM})$ was included in all of the buffers to minimize oxidation. All solutions were purged and capped with nitrogen to simulate the anoxic condition of midgut fluid.

Fifty microliters of a tannic acid solution (10.0 $\mathrm{mg} / \mathrm{ml}$ double-distilled water) was mixed with 450 $\mu \mathrm{l}$ of each buffer (5 replicates of each), and after a 30 -min incubation period $\left(22^{\circ} \mathrm{C}\right)$ any precipitate that formed was removed by centrifugation $(13,600 \mathrm{~g}, 10$ or $\left.15 \mathrm{~min}, 22^{\circ} \mathrm{C}\right)$. An aliquot $(50 \mu \mathrm{l})$ of each supernatant solution was mixed with $450 \mu \mathrm{l}$ of $90 \%$ acetonitrile, filtered $(0.45 \mu$, GHP, Gelman Sciences, Ann Arbor, MI) into HPLC vials and analyzed for tannic acid, as described below. Pellets were re-solubilized in $500 \mu \mathrm{l}$ of 0.05 M HEPES buffer ( $\mathrm{pH} 6.5$ ), and a $50-\mu \mathrm{l}$ aliquot of the solution was mixed with $450 \mu \mathrm{l}$ of $90 \%$ acetonitrile, filtered into HPLC vials and analyzed for tannic acid, as described below.

\section{Removal of Divalent Cations, Proteins, and Lipids From Gut Fluid}

Divalent cations were removed from gut fluid using the chelating resin, Chelex 100 (Sigma Chemical Co.). Aliquots $(65 \mu \mathrm{l})$ of gut fluid were mixed with Chelex (9.7-11.4 mg) in screw-cap centrifuge tubes $(2.0 \mathrm{ml})$ under nitrogen and shaken for $15 \mathrm{~min}$. Gut fluid was separated from the Chelex by centrifugation $(13,600 \mathrm{~g}, 5 \mathrm{~min})$. Chelextreated samples of gut fluid were prepared from larvae that had been reared entirely on an artificial diet, as well as larvae that had been switched from artificial diet to tomato leaves during the first two days of the final instar. Gut fluid was deproteinized by mixing an aliquot $(840 \mu \mathrm{l})$ with 
9 volumes of nitrogen-purged ethanol (30 min, $4^{\circ} \mathrm{C}$ ) (Martin and Martin, 1984) in a screw-cap centrifuge tube flushed with nitrogen. After centrifugation $\left(1,800 \mathrm{~g}, 4^{\circ} \mathrm{C}\right)$, the supernatant solution was transferred to test tubes, and ethanol was evaporated under a stream of nitrogen. Double-distilled water $(478 \mu \mathrm{l})$ was added to the deproteinized gut fluid to return it to its original volume. Lipids were removed from the gut fluid by extracting an aliquot $(425 \mu \mathrm{l})$ with a mixture of methanol $(566 \mu \mathrm{l})$ and methylene dichloride $(1,130 \mu \mathrm{l})$ in a screw-cap centrifuge tube $(2.0 \mathrm{ml})$ in a shaker $\left(10 \mathrm{~min}, 22^{\circ} \mathrm{C}\right)$. All solvents were first purged with nitrogen. The mixture was separated into two phases by gentle centrifugation (133g, 3 min, $22^{\circ} \mathrm{C}$ ), after which the upper phase (water and methanol) was pipetted into a centrifuge tube and extracted twice with methylene dichloride $(1,130 \mu \mathrm{l})$. Methanol was evaporated under a stream of nitrogen, and double-distilled water $(210 \mu \mathrm{l})$ was added to the defatted gut fluid to return it to its original volume.

\section{Interaction of Tannic Acid With Gut Fluid}

Five microliters of a solution of tannic acid $(10.0 \mathrm{mg} / \mathrm{ml}$ double-distilled water) that had been purged with nitrogen was mixed with $45 \mu$ l of treated or untreated gut fluid in a $2.0-\mathrm{ml}$ screwcap centrifuge tube ( 7 replicates). The tube was capped with nitrogen and incubated for $30 \mathrm{~min}$ at $22^{\circ} \mathrm{C}$, after which the tube was centrifuged $\left(12,000 \mathrm{~g}, 4.7^{\circ} \mathrm{C}, 15 \mathrm{~min}\right)$ and then examined for the presence of a precipitate. An 8- $\mu$ l aliquot of each supernatant solution was mixed with 192 $\mu \mathrm{l}$ of $56.5 \%$ acetonitrile containing $0.5 \%$ acetic acid, filtered (Gelman GHP, $0.45 \mu \mathrm{m}$ ) into an HPLC vial, flushed with nitrogen, and analyzed for tannic acid, as described below.

\section{Ultrafiltration of Supernatant Solutions From Buffers and Gut Fluid Samples Treated With Tannic Acid}

Aliquots of each of the supernatant solutions from the experiments described above $(20-30 \mu \mathrm{l}$ for the experiments using gut fluid, $50 \mu \mathrm{l}$ for the experiments using buffers) were placed in ultrafilters (Ultrafree-0.5, Millipore Corp., Bedford, MA) with a molecular weight cutoff (MWCO) of 100,000 Daltons. The ultrafilters were flushed with nitrogen and then centrifuged $\left(10,000 \mathrm{~g}, 4.7^{\circ} \mathrm{C}, 30 \mathrm{~min}\right.$, for experiments using midgut fluid, $7 \mathrm{~min}$ for experiments using buffers). The volumes of the ultrafiltrates were measured using an adjustable 200- $\mu$ l Gilson pipette; the volumes of the retentates
$(3.2 \pm 0.4 \mu \mathrm{l}$ in experiments with buffers; $3.0 \pm 0.2$ $\mu \mathrm{l}$ in experiments with gut fluid), using a $10-\mu \mathrm{l}$ Hamilton microsyringe. An aliquot of the ultrafiltrate $(8 \mu \mathrm{l}$ for ultrafiltrates from experiments with gut fluid, $50 \mu \mathrm{l}$ for ultrafiltrates from experiments with buffers) and the entire retentate were each mixed with acidified $56.5 \%$ acetonitrile $(450 \mu \mathrm{l}$ in experiments with gut fluid, 195-225 $\mu \mathrm{l}$ in experiments with buffers), filtered (Gelman GHP, $0.45 \mu \mathrm{m}$ ) into an HPLC vial that was flushed with nitrogen, and analyzed for tannic acid, as described below. To determine whether any tannic acid remained adsorbed on the ultrafilter membrane, $100 \mu \mathrm{l}$ of acidified 56\% acetonitrile was placed in the ultrafilter, the device was centrifuged $\left(10,000 \mathrm{~g}, 4.7^{\circ} \mathrm{C}\right.$, $30 \mathrm{~min}$ ), and a $50-\mu \mathrm{l}$ aliquot of the resulting ultrafiltrate was mixed with $450 \mu \mathrm{l}$ of acidified $56 \%$ acetonitrile and analyzed for tannic acid, as described below. The percentage of tannic acid placed in the ultrafilter that was adsorbed to the ultrafilter membrane during these experiments was $0.7 \pm$ $0.4,0.6 \pm 0.2,3.5 \pm 0.8$, and $11-21 \%$ from the buffer solution, the monovalent cation solution, the divalent cation solution, and samples of midgut fluid, respectively.

\section{Chemical Analyses}

Tannic acid was assayed using reverse-phase high-performance liquid chromatography (HPLC). Aliquots $(20$ or $25 \mu \mathrm{l}$ ) of test solutions were injected onto a Vydac C-18 column $(5 \mu \mathrm{m}, 250 \times 4.6$ $\mathrm{mm}$ ) and guard column using a Shimadzu autoinjector. The components of tannic acid (galloyl glucose esters) were eluted with a mobile phase of $23 \%(\mathrm{v} / \mathrm{v})$ aqueous acetonitrile, containing $1 \%$ acetic acid, and were detected at $280 \mathrm{~nm}(0.002$ AUFS) with a Shimadzu uv-visible detector. Appropriate controls were run to determine whether interfering substances were present, and peak areas of test samples were corrected accordingly. Peak areas were integrated with a Shimadzu CR4A Chromatopac computer. Standard curves were made for tannic acid to convert peak areas to $\mu \mathrm{g}$ injected. The values reported for tannic acid represent only the galloyl glucose esters and do not include the gallic acid present in small amounts in the commercial tannic acid preparation used in these experiments.

\section{Statistical Analyses}

Statistical comparisons were made between means within experiments to avoid any confounding effects from variation in methods between experiments. Pairwise comparisons of means were 
made using Mann-Whitney U-tests with SYSTAT (Wilkinson, 1990). When multiple pairwise comparisons were made, the level of significance was adjusted by the number of tests performed $(\alpha / \mathrm{K})$ (Rice, 1989). Comparisons of three means were made with Kruskal-Wallis tests.

\section{RESULTS}

\section{Concentrations of Monovalent and Divalent Cations in Gut Fluid}

The concentrations of sodium, potassium, magnesium, calcium, and iron in supernatant solutions from centrifuged samples of midgut fluid obtained from $M$. sexta and O. leucostigma larvae are summarized in Table 1 . The values for sodium and potassium that we measured in $M$. sexta and $O$. leucostigma are roughly comparable to the values reported in Philosamia cynthia (1.0 \pm 0.2 and $196.8 \pm 7.1 \mathrm{mM}$, respectively) and Bombyx mori $(1.3 \pm 0.1$ and $149.5 \pm 2.9 \mathrm{mM}$, respectively) (Giordana and Sacchi, 1978). However, the levels of magnesium and calcium that we measured in $M$. sexta and $O$. leucostigma midgut fluids are much lower than those reported by Giordana and Sacchi (1978): P. cynthia, $8.6 \pm 0.4$ and $11.0 \pm 1.0 \mathrm{mM}$, respectively; $B$. mori, $29.4 \pm$ 3.4 and $19.6 \pm 2.0 \mathrm{mM}$, respectively.

\section{Interaction of Tannic Acid With Monovalent and Divalent Cations}

A white precipitate formed immediately upon the addition of a tannic acid solution to solutions containing $21 \mathrm{mM}$ magnesium chloride and 32 $\mathrm{mM}$ calcium chloride at $\mathrm{pH} 8.0$ and 10.0. The precipitates contained 63 and $83 \%$ of the tannic acid originally added to the divalent-cation containing solutions at $\mathrm{pH} 8.0$ and 10.0 , respectively, whereas the supernatant solutions obtained by centrifuging these mixtures contained only 15 and $4 \%$ of the original tannic acid (Table 2). No precipitate was formed when tannic acid was added to these divalent cation-containing solutions at $\mathrm{pH}$ 6.5. Neither was any visible precipitate formed at any $\mathrm{pH}$ when tannic acid was added to buffered solutions containing $3 \mathrm{mM}$ sodium chloride and $160 \mathrm{mM}$ potassium chloride, or to buffer solutions at $\mathrm{pH} 8.0$ and 10.0 containing 0.51 and $0.33 \mathrm{mM}$ magnesium and calcium chloride, respectively, in the presence or absence of 2.6 and $153.8 \mathrm{mM}$ sodium and potassium chloride. In those experiments conducted under conditions that did not result in precipitate formation, 77$100 \%$ of the tannic acid added initially to the buffer solution was still detectable in the solution at the end of the incubation period (Table 2). Oxidation is probably responsible for recoveries of tannic acid less than $100 \%$.

\section{Interaction of Tannic Acid With M. sexta Gut Fluid}

Although addition of tannic acid to the midgut fluid of $M$. sexta larvae fed on tomato foliage did not produce a copious precipitate, centrifugation separated the gut fluid-tannic acid mixture into a green pellet and a supernatant layer that contained $65-72 \%$ of the tannic acid originally added to the gut fluid (Table 3). When tannic acid was added to deproteinized or defatted gut fluid, or to gut fluid treated with Chelex 100 (to remove magnesium and calcium ions), no visible pellet was produced and a higher percentage (88-96\%) of the tannic acid initially added to the gut fluid sample remained in the supernatant layer (Table 3). No pellet was formed upon centrifugation of gut fluid to which no tannic acid had been added.

Similar results were obtained when tannic acid was mixed with midgut fluid obtained from $M$. sexta larvae that had fed on artificial diet. When untreated gut fluid was mixed with tannic acid, $76.9 \pm 6.5 \%$ of the added tannic acid remained in the supernatant layer, whereas when Chelex-treated gut fluid was mixed with tannic acid, virtually all $(100.4 \pm 12.0 \%)$ of the added tannic acid remained in the supernatant layer.

\section{Ultrafiltration of Tannic Acid in Supernatant Layers}

The tannic acid in the supernatant layers obtained by centrifuging a mixture of gut fluid and tannic acid was concentrated in the retentate

TABLE 1. Concentrations (mM) of Sodium, Potassium, Magnesium, Calcium and Iron in Supernatant Solutions From Centrifuged Samples of Midgut Fluid Obtained From $M$. sexta and $O$. leucostigma larvae*

\begin{tabular}{|c|c|c|c|c|c|c|}
\hline Species & Diet & Sodium & Potassium & Magnesium & Calcium & Iron \\
\hline$M$. & Tomato foliage & $2.0 \pm 0.02$ & $157.2 \pm 15.9$ & $0.50 \pm 0.03$ & $0.37 \pm 0.16$ & $0.072 \pm 0.004$ \\
\hline M. sexta & Artificial diet & $4.6 \pm 1.52$ & $129.5 \pm 0.50$ & $0.31 \pm 0.01$ & $0.19 \pm 0.02$ & $0.037 \pm 0.003$ \\
\hline O. leucostigma & Elm foliage & $1.4 \pm 0.12$ & $168.5 \pm 18.5$ & $1.19 \pm 0.19$ & $0.82 \pm 0.12$ & $0.057 \pm 0.007$ \\
\hline O. leucostigma & Artificial diet & $3.7 \pm 1.7$ & $139.0 \pm 13.6$ & $0.35 \pm 0.06$ & $0.16 \pm 0.05$ & $0.039 \pm 0.006$ \\
\hline
\end{tabular}

*Data reported as mean $\pm \mathrm{SE}$. 
TABLE 2. Tannic Acid Present in the Precipitates and the Supernatant Solutions Obtained by Centrifuging Mixtures Prepared by Adding Tannic Acid $(0.48 \mathrm{mg} \text { in } 50 \mu \mathrm{l})^{\mathrm{a}}$ to $450 \mu \mathrm{l}$ of Buffer $(\mathrm{HEPES}$, pH 6.5 and 8.0, and Glycine, pH 10.0)*

\begin{tabular}{|c|c|c|c|c|c|c|}
\hline \multirow[b]{2}{*}{$\mathrm{pH}$} & \multicolumn{4}{|c|}{ Ion concentrations (mM) } & \multicolumn{2}{|c|}{ Tannic acid $(\mathrm{mg})^{\mathrm{a}}$} \\
\hline & $\mathrm{Na}$ & $\mathrm{K}$ & $\mathrm{Mg}$ & $\mathrm{Ca}$ & In precipitate ${ }^{3}$ & In supernatant \\
\hline 6.5 & 0 & 0 & 0 & 0 & 0 & $0.44 \pm 0.01$ \\
\hline 6.5 & 3 & 160 & 0 & 0 & 0 & $0.40 \pm 0.01$ \\
\hline 6.5 & 0 & 0 & 21 & 32 & 0 & $0.40 \pm 0.005$ \\
\hline 8.0 & 0 & 0 & 0 & 0 & 0 & $0.39 \pm 0.005$ \\
\hline 8.0 & 3 & 160 & 0 & 0 & 0 & $0.37 \pm 0.01$ \\
\hline 8.0 & 0 & 0 & 21 & 32 & $0.30 \pm 0.03$ & $0.06 \pm 0.001$ \\
\hline 10.0 & 0 & 0 & 0 & 0 & 0 & $0.46 \pm 0.01$ \\
\hline 10.0 & 3 & 160 & 0 & 0 & 0 & $0.45 \pm 0.02$ \\
\hline 10.0 & 0 & 0 & 21 & 32 & $0.40 \pm 0.01$ & $0.02 \pm 0.002$ \\
\hline 8.0 & 0 & 0 & 0.51 & 0.33 & 0 & $0.49 \pm 0.009$ \\
\hline 8.0 & 2.6 & 153.8 & 0.51 & 0.33 & 0 & $0.41 \pm 0.01$ \\
\hline 10.0 & 0 & 0 & 0.51 & 0.33 & 0 & $0.41 \pm 0.007$ \\
\hline 10.0 & 2.6 & 153.8 & 0.51 & 0.33 & 0 & $0.43 \pm 0.007$ \\
\hline
\end{tabular}

*Data reported as mean $\pm \mathrm{SE}(\mathrm{N}=5)$.

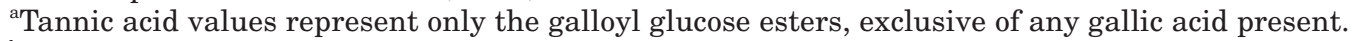

${ }^{\mathrm{b}}$ An entry of zero means that there was no visible precipitate formed when the tannic acid solution was added to the buffer and that no visible pellet was produced by centrifugation.

during ultrafiltration. Retentates from the mixtures of tannic acid and untreated, Chelextreated, or defatted gut fluid had tannic acid concentrations 2.0-2.9 times higher than the ultrafiltrates and 1.7-2.1 times higher than the original solutions (Table 4). Although we did not measure the concentration of tannic acid in the retentate from deproteinized gut fluid containing tannic acid, the fact that the concentration in the ultrafiltrate was lower than the concentration in the original solution strongly implies that tannic acid was concentrated in this retentate as well.

In contrast to the tannic acid remaining in the supernatant layer from gut fluid-tannic acid mixtures, tannic acid remaining in the supernatant layer from $\mathrm{pH} 10$ buffer-tannic acid mixtures

TABLE 3. Percent of tannic acid remaining in supernatant layer following centrifugation of a mixture prepared by adding tannic acid $(48 \mu \mathrm{g}$ in $5 \mu \mathrm{l})$ to $45 \mu \mathrm{l}$ of untreated or treated gut fluid*

Tannic acid in supernatant solution Gut fluid (\% of amount originally present)

\begin{tabular}{ll} 
Untreated $^{\mathrm{a}}$ & $68.7 \pm 4.6^{\mathrm{b}}$ \\
Deproteinized & $91.2 \pm 4.8^{\mathrm{c}}$ \\
Defatted & $88.3 \pm 5.0^{\mathrm{c}}$ \\
Chelex-treated & $95.6 \pm 7.2^{\mathrm{c}}$ \\
\hline
\end{tabular}

*Tannic acid values represent only the galloyl glucose esters, exclusive of any gallic acid present. Data reported as mean $\pm \mathrm{SE}(\mathrm{N}=7)$. Values followed by a different letter are significantly different $(P<0.05)$.

${ }^{a}$ Means from the separate controls (untreated) for the three treatments were pooled to give an overall mean $(\mathrm{N}=3)$. could not be concentrated by ultrafiltration. In none of the three treatments was the tannic acid concentration in the retentate significantly higher than in either the ultrafiltrate or the original solution (Table 4).

\section{DISCUSSION}

The precipitation and complexation of polyphenols by polyvalent cations in simple aqueous solutions is well known from the work of earlier investigators (Murdiati et al., 1991; Haslam et al., 1992; Slabbert, 1992; McDonald et al., 1996). In this study, we have established that magnesium and calcium ions, in concentrations roughly comparable to those reported in the midguts of two species of herbivorous lepidopteran larvae (Giordana and Sacchi, 1978), bring about the precipitation of most of the tannic acid present in simple solutions buffered at $\mathrm{pH} 8.0$ and $10.0, \mathrm{pHs}$ that fall within the range of 8-12 commonly reported for the midguts of lepidopteran larvae (Berenbaum, 1980; Dow, 1986). pHs of 8.0-9.3 have been reported in the midgut of $M$. sexta (Appel and Martin, 1990). The precipitate is readily centrifuged into a pellet, and the tannic acid that is not precipitated readily passes through an ultrafiltration membrane with a molecular weight cutoff of 100,000 Daltons. This demonstrates that the tannic acid that remains in the supernatant layer is in true solution, either as a monomer or as an oligomeric complex with molecular dimensions that do not exceed those of the 
TABLE 4. Tannic Acid Concentrations in Retentates and Ultrafiltrates From Supernatant Solutions Obtained by Centrifuging Mixtures of Tannic Acid and M. sexta gut Fluid or Tannic Acid and Glycine Buffers (pH 10.0)*

\begin{tabular}{|c|c|c|c|}
\hline \multirow[b]{2}{*}{ Origin of supernatant layer } & \multicolumn{3}{|c|}{ Tannic acid concentration $(\mu \mathrm{g} / \mu \mathrm{l})^{\mathrm{a}}$} \\
\hline & Original supernatant & Retentate & Ultrafiltrate \\
\hline Untreated gut fluid $* *$ & $0.77 \pm 0.06^{\mathrm{d}}$ & $0.96 \pm 0.05^{\mathrm{d}}$ & $0.48 \pm 0.03^{\mathrm{e}}$ \\
\hline Chelex-treated gut fluid & $1.13 \pm 0.09^{\mathrm{d}}$ & $1.55 \pm 0.12^{\mathrm{e}}$ & $0.53 \pm 0.02^{\mathrm{f}}$ \\
\hline Deproteinized gut fluid & $1.12 \pm 0.14^{\mathrm{d}}$ & ND & $0.63 \pm 0.05^{\mathrm{e}}$ \\
\hline Defatted gut fluid** & $0.88 \pm 0.05^{\mathrm{d}}$ & $1.89 \pm 0.23^{\mathrm{e}}$ & $0.75 \pm .0 .04^{\mathrm{d}}$ \\
\hline Buffer & $0.69 \pm 0.03^{\mathrm{d}}$ & $0.59 \pm 0.03^{\mathrm{d}}$ & $0.67 \pm 0.02^{\mathrm{d}}$ \\
\hline Buffer plus $\mathrm{NaCl}$ and $\mathrm{KCl}^{\mathrm{b}}$ & $0.75 \pm 0.02^{\mathrm{d}}$ & $0.62 \pm 0.05^{\mathrm{d}}$ & $0.71 \pm 0.04^{\mathrm{d}}$ \\
\hline Buffer plus $\mathrm{MgCl}_{2}$ and $\mathrm{CaCl}_{2}{ }^{\mathrm{c}}$ & $0.072 \pm 0.010^{\mathrm{d}}$ & $0.075 \pm 0.009^{\mathrm{d}}$ & $0.073 \pm 0.009^{\mathrm{d}}$ \\
\hline
\end{tabular}

"The ultrafiltration membrane had a MWCO of 100,000 Daltons. Data reported as mean \pm SE. Values within a row followed by a different letter are significantly different $(P<0.05)$. ND = not determined.

**The differences between tannic acid concentrations in original supernatant and retentate in the experiment with untreated gut fluid and original supernatant and ultrafiltrate in the experiment with defatted gut fluid approach significance ( $P=0.087$ and $P=0.084$, respectively).

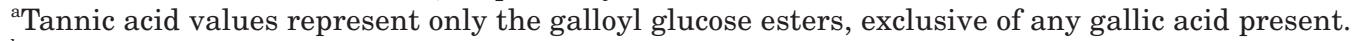

b $3 \mathrm{mM}$ sodium chloride, $160 \mathrm{mM}$ potassium chloride.

${ }^{\mathrm{c}} 21 \mathrm{mM}$ magnesium chloride, $32 \mathrm{mM}$ calcium chloride.

pores in the ultrafiltration membrane (approximately $5 \mathrm{~nm}$ ).

On the other hand, magnesium and calcium ions do not precipitate tannic acid from aqueous solutions at $\mathrm{pH} 8.0$ and 10.0 when these ions are present at the low concentrations that we measured in the midgut fluids of $M$. sexta and $O$. leucostigma. We suspect that more efficient centrifugation during sample preparation is the most likely explanation for why we found much lower levels of magnesium and calcium ions in our samples of midgut fluid that Giordana and Sacchi (1978) found in theirs. Thus, our values represent only the ions present in true solution, whereas the values reported by Giordana and Sacchi probably include significant quantities of magnesium and calcium ions that are suspended in the gut milieu. In support of this interpretation, we note that the ratios of alkaline earth ions (magnesium and calcium) to alkali metal ions (sodium and potassium) are much closer to the ratios of these ions in foliage in the Giordana and Sacchi (1978) samples than in ours. The average value of the ratio of alkaline earth to alkali metal ions in 39 species of tree, shrub and herb foliage was 0.83 ( $\mathrm{SD}=0.50)$ (Allen, 1974). Giordana and Sacchi (1978) reported values of 0.10 and 0.32 in $P$. cynthia and B. mori, respectively, whereas we found ratios one or two orders of magnitude lower. We conclude, therefore, that the higher values reported by Giordana and Sacchi (1978) provide a more realistic estimate than ours of the total amounts of divalent alkaline earth ions available for binding with tannins or other midgut constitu- ents with anionic binding sites, since our values represent only the portions of these ions that remain in true solution.

In contrast to the extensive precipitate that forms when tannic acid is added to a simple buffer solution containing $21 \mathrm{mM}$ magnesium chloride and $32 \mathrm{mM}$ calcium chloride, a smaller amount of precipitate is produced when tannic acid is added to $M$. sexta gut fluid. Less than $31 \%$ of the tannic acid originally added to the gut fluid can be centrifuged into a pellet, and as much as $80 \%$ of the tannic acid that remains in the supernatant layer following centrifugation is retained by an ultrafiltration membrane (MWCO 100,000 Daltons). This demonstrates that little of the tannic acid in $M$. sexta gut fluid is in true solution. Some tannic acid precipitates; most exists in the form of a colloidal suspension. Under the alkaline conditions of a caterpillar's gut ( $\mathrm{pH} 8-12$ ) (Berenbaum, 1980; Dow, 1986) tannins would occur largely as polyanions, which can form complexes with divalent cations (Martin et al., 1985; Murdiati et al., 1991; Haslam et al., 1992; Slabbert, 1992; McDonald et al., 1996), surface-active phospholipids (DeVeau and Schultz, 1992), and some proteins (Martin et al., 1985; Hagerman and Klucher, 1986; Haslam et al., 1992; Stern et al., 1996). Thus, the insoluble tannin-containing phase that is produced when tannic acid is mixed with gut fluid is probably a complex multi-molecular aggregate of indefinite chemical composition. It may also contain polymers formed by chemical reactions between midgut constituents and quinoidal oxidation products of the tannic 
acid (Felton et al., 1989; Appel, 1993; Summers and Felton, 1994; Stern et al., 1996). The formation of a tannin-containing colloidal phase when tannic acid is added to gut fluid that has been treated to remove proteins, lipids, or divalent cations attests to the chemically undefined nature of the insoluble tannic acid-containing aggregate that is produced.

Earlier efforts to explain the impermeability of the PEs of lepidopteran larvae to tannins have invoked special properties of the PE, e.g., the presence of substances in the matrix that strongly adsorb polyphenols (Bernays, 1981) or the presence of an extensive network of fixed anionic sites in the PE matrix that acts as an electrostatic barrier to the passage of polyphenolate anions (Barbehenn and Martin, 1994). Neither of these explanations has survived rigorous testing (Barbehenn and Martin, 1992, 1997). On the basis of the present study, we suggest that the failure of tannins to diffuse across the PEs of lepidopteran larvae is a result of the physical state of tannins in the gut milieu rather than any special permeability properties of the matrix of the PE. We conclude that tannins either precipitate in a caterpillar's gut or form high molecular weight multi-component colloidal aggregates, and that the failure of tannins to cross the PE is due largely to the capacity of the PE to act as a physical barrier to insoluble and colloidally dispersed particles.

This interpretation of the failure of tannins to cross the PEs of larval Lepidoptera also provides a possible rationale for the observation that tannic acid does diffuse across the PEs of some grasshoppers (Barbehenn et al., 1996). The guts of grasshoppers are acidic or near neutrality, with pHs in the range 5.5-7.4 (Ferreira et al., 1990; Barbehenn et al., 1996). As we have shown in this study, tannic acid is not precipitated by magnesium and calcium ions at $\mathrm{pH}$ 6.5. Although we cannot predict the effect of the lower $\mathrm{pH}$ on all of the many equilibria between tannic acid and the various midgut constituents that can bind with it, it is possible that in Orthoptera not all of the tannic acid is bound up in insoluble or colloidally dispersed multi-molecular complexes. Some may exist in true solution. Thus, to the extent that the $\mathrm{PE}$ is an effective barrier only to the tannic acid present in an insoluble precipitate or in colloidal form, and not to tannic acid in true solution, some tannic acid would be expected to diffuse across the PEs of Orthoptera.

\section{ACKNOWLEDGMENTS}

This study was supported by NSF grant BSR-8904043 to M.M.M. and R.V.B. We thank Dr. Ted Huston for carrying out the measurements of cation concentrations in gut fluid samples.

\section{LITERATURE CITED}

Allen SE (1974): Chemical composition of soils and plant materials. In: Allen SE (ed): Analysis of ecological materials. Oxford: Blackwell, pp 503-511.

Appel HM (1993): Phenolics in ecological interactions: the importance of oxidation. J Chem Ecol. 19:1521-1552.

Appel HM, Martin MM (1990): Gut redox conditions in herbivorous lepidopteran larvae. J. Chem Ecol 16:32773290 .

Barbehenn RV, Martin MM (1992): Protective role of the peritrophic membrane in the tannin-tolerant larvae of Orgyia leucostigma (Lymantriidae). J Insect Physiol 38:973-980.

Barbehenn RV, Martin MM (1994): Tannin sensitivity in the larvae of Malacosoma disstria (Lepidoptera): roles of the peritrophic envelope and midgut oxidation. J Chem Ecol 20:1985-2001.

Barbehenn RV, Martin MM (1995): Peritrophic envelope permeability in herbivorous insects. J Insect Physiol 41:303-311.

Barbehenn RV, Martin MM (1997): Permeability of the peritrophic envelopes of herbivorous insects to dextran sulfate: a test of the polyanion exclusion hypothesis. J Insect Physiol 43:243-249.

Barbehenn RV, Martin MM, Hagerman AE (1996): Reassessment of the roles of the peritrophic envelope and hydrolysis in protecting polyphagous grasshoppers from ingested hydrolyzable tannins. J Chem Ecol 22:1901-1919.

Baxter NJ, Lilley TH, Haslam E., Williamson MP (1997): Multiple interactions between polyphenols and a salivary proline-rich protein repeat result in complexation and precipitation. Biochemistry 36:5566-5577.

Berenbaum MR (1980): Adaptive significance of midgut $\mathrm{pH}$ in larval Lepidoptera. Am Nat 115:138-146.

Bernays EA (1981): Plant tannins and insect herbivores: an appraisal. Ecol Entomol 6:353-360.

Cai Y, Gaffney SH, Lilley TH, Haslam E (1989): Carbohydratepolyphenol complexation. In: Hemingway RW, Karchesy JJ (eds): Chemistry and significance of condensed tannins. New York: Plenum Press, pp 307-322.

Cai Y, Gaffney SH, Lilley TH, Magnolato D, Martin R., Spencer CM., Haslam E (1990): Polyphenol interactions. 
Part 4. Model studies with caffeine and cyclodextrins. J Chem Soc Perkin Trans 2:2197-2209.

DeVeau EJI, Schultz JC (1992): Reassessment of interaction between gut detergents and tannins in Lepidoptera and significance for gypsy moth larvae. J Chem Ecol 8:1437-1453.

Derksen ACG, Granados RR (1988): Alteration of a lepidopteran peritrophic membrane by baculoviruses and enhancement of viral infectivity. Virology 167: $242-250$.

Dow JAT (1986): Insect midgut function. Adv Insect Physiol 19:187-328.

Feeny P (1970): Seasonal changes in oak leaf tannins and nutrients as a cause of spring feeding by winter moth caterpillars. Ecology 51:565-581.

Felton GW, Donato K, Del Vecchio RJ, Duffey SS (1989): Activation of plant polyphenol oxidases by insect feeding reduces the nutritive quality of foliage for noctuid herbivores. J Chem Ecol 15:2667-2694.

Ferreira C, Oliveira MC, Terra WR (1990): Compartmentalization of the digestive process in Abracris flavolineata Orthoptera: Acrididae) adults. Insect Biochem 20:267-274.

Ferreira C, Capella AN, Sitnik R, Terra WR (1994): Properties of the digestive enzymes and the permeability of the peritrophic membrane of Spodoptera frugiperda (Lepidoptera) larvae. Comp Biochem Physiol 107A:631-640.

Giordana B, Sacchi F (1978): Cellular ionic concentrations in the midgut of two larvae of Lepidoptera in vivo and in vitro. Comp Biochem Physiol 59A:17-20.

Hagerman AE, Klucher KM (1986): Tannin-protein interactions. In: Cody V, Middleton E, Harborne JB (eds): Plant flavonoids in biology and medicine: biochemical, pharmacological, and structure-activity relationships. New York: Liss, pp 67-76.

Haslam E. Lilley TH, Warminski E, Liao H, Cai Y, Martin R, Gaffney SH, Goulding PN, Luck G (1992): Polyphenol complexation: a study in molecular recognition. In: Ho CT, Lee CY, Huang MT (eds): Phenolic compounds in food and their effects on health I: analysis, occurrence, and chemistry. Washington, DC: American Chemical Society, ACS Symposium Series 506, pp 8-50.

Ikeda I, Imasato Y, Sasaki E, Nakayama M, Nagao H, Takeo T, Yayabe F, Sugano M (1992): Tea catechins decrease micellar solubility and intestinal absorption of cholesterol in rats. Biochim Biophys Acta 1127:141-146.

Martin MM, Martin JS (1984): Surfactants: their role in preventing the precipitation of proteins by tannins in insect guts. Oecologia 61:342-345.

Martin MM, Rockholm DC, Martin JS (1985): Effects of surfactants, $\mathrm{pH}$, and certain cations on precipitation of proteins by tannins. J Chem Ecol 11:485-494.

McDonald M, Mila I, Scalbert A (1996): Precipitation of metal ions by plant polyphenols: Optimal conditions and origin of precipitation. J Agric Food Chem 44:599-606.

Murdiati TB, McSweeney CS, Lowry JB (1991): Complexing of toxic hydrolyzable tannins of yellow-wood (Terminalia oblongata) and harendong (Clidemia hirta) with reactive substances: an approach to preventing toxicity. J Appl Toxicol 11:333-338.

Peters W (1992): Peritrophic membranes. Berlin: Springer.

Peters W, Wiese B (1986): Permeability of the peritrophic membranes of some Diptera to labeled dextrans. J Insect Physiol 32:43-50.

Rice WR (1989): Analyzing tables of statistical tests. Evolution 43:223-225.

Santos CD, Terra WR (1986): Distribution and characterization of oligomeric digestive enzymes from Erinnyis ello larvae and inferences concerning secretory mechanisms and permeability of the peritrophic membrane. Insect Biochem 16:691-700.

Slabbert N (1992): Complexation of condensed tannins with metal ions. In: Hemingway RW, Laks PE (eds): Plant polyphenols. New York: Plenum Press, pp 421-436.

Stern JL, Hagerman AE, Steinberg PD, Mason PK (1996): Phlorotannin-protein interactions. J Chem Ecol 22: 1877-1899.

Summers CB, Felton GW (1994): Prooxidant effects of phenolic acids on the generalist herbivore Helicoverpa zea (Lepidoptera: Noctuidae): potential mode of action for phenolic compounds in plant anti-herbivore chemistry. Insect Biochem Mol Biol 24:943-953.

Takechi M, Tanaka Y (1987): Binding of 1,2,3,4,6-pentagalloylglucose to proteins, lipids, nucleic acids and sugars. Phytochemistry 26:95-97.

Wilkinson L. (1990): SYSTAT: the system for statistics. Evanston, IL: SYSTAT Inc. 